\title{
TRIANGULUM II: POSSIBLY A VERY DENSE ULTRA-FAINT DWARF GALAXY*
}

\author{
Evan N. Kirby ${ }^{1}$, Judith G. Cohen ${ }^{1}$, Joshua D. Simon ${ }^{2}$, and Puragra Guhathakurta ${ }^{3}$ \\ ${ }^{1}$ California Institute of Technology, 1200 E. California Boulevard, MC 249-17, Pasadena, CA 91125, USA \\ ${ }^{2}$ Observatories of the Carnegie Institution of Washington, 813 Santa Barbara Street, Pasadena, CA 91101, USA \\ ${ }^{3}$ UCO/Lick Observatory and Department of Astronomy and Astrophysics, University of California, 1156 High Street, Santa Cruz, CA 95064, USA \\ Received 2015 October 12; accepted 2015 November 3; published 2015 November 16
}

\section{ABSTRACT}

Laevens et al. recently discovered Triangulum II (Tri II), a satellite of the Milky Way. Its Galactocentric distance is $36 \mathrm{kpc}$, and its luminosity is only $450 L_{\odot}$. Using Keck/DEIMOS, we measured the radial velocities of six member stars within $1 ! 2$ of the center of Tri II, and we found a velocity dispersion of $\sigma_{v}=5.1_{-1.4}^{+4.0} \mathrm{~km} \mathrm{~s}^{-1}$. We also measured the metallicities of three stars and found a range of $0.8 \mathrm{dex}$ in $[\mathrm{Fe} / \mathrm{H}]$. The velocity and metallicity dispersions identify Tri II as a dark matter-dominated galaxy. The galaxy is moving very quickly toward the Galactic center $\left(v_{\mathrm{GSR}}=-262 \mathrm{~km} \mathrm{~s}^{-1}\right)$. Although it might be in the process of being tidally disrupted as it approaches pericenter, there is no strong evidence for disruption in our data set. The ellipticity is low, and the mean velocity, $\left\langle v_{\text {helio }}\right\rangle=-382.1 \pm 2.9 \mathrm{~km} \mathrm{~s}^{-1}$, rules out an association with the Triangulum-Andromeda substructure or the Pan-Andromeda Archaeological Survey stellar stream. If Tri II is in dynamical equilibrium, then it would have a mass-to-light ratio of $3600_{-2100}^{+3500} M_{\odot} L_{\odot}^{-1}$, the highest of any non-disrupting galaxy (those for which dynamical mass estimates are reliable). The density within the $3 \mathrm{D}$ half-light radius would be $4.8_{-3.5}^{+8.1} M_{\odot} \mathrm{pc}^{-3}$, even higher than Segue 1. Hence, Tri II is an excellent candidate for the indirect detection of dark matter annihilation.

Key words: galaxies: abundances - galaxies: dwarf - Local Group

\section{INTRODUCTION}

The Sloan Digital Sky Survey (SDSS; Abazajian et al. 2009) revolutionized Local Group astronomy in the last decade by discovering more than a dozen new dwarf galaxies around the Milky Way (MW). We are now in the midst of another revolution. The Panoramic Survey Telescope and Rapid Response System (Pan-STARRS; Kaiser et al. 2010), the Dark Energy Survey (DES, Flaugher et al. 2012), and other Dark Energy Camera imaging surveys have discovered more than 20 previously unknown MW satellites (e.g., Bechtol et al. 2015; Kim \& Jerjen 2015; Laevens et al. 2015a). The greater photometric depth and expanded sky coverage of PanSTARRS and DES over SDSS has enabled the discovery of many new satellites with luminosities less than $10^{4} L_{\odot}$ and also satellites more distant than $200 \mathrm{kpc}$.

Dwarf galaxy candidates are discovered through imaging, but their identification as galaxies or star clusters is made secure through spectroscopy (e.g., Willman \& Strader 2012). A candidate can be considered a galaxy if it shows evidence for dark matter, including a velocity dispersion in excess of what would be expected from stellar mass alone or a dispersion in stellar metallicity, which indicates chemical self-enrichment. Spectroscopy of satellites discovered in the last two years has already confirmed five new galaxies and one globular cluster (Kirby et al. 2015b; Koposov et al. 2015; Martin et al. 2015; Simon et al. 2015; Walker et al. 2015).

Laevens et al. (2015b) discovered Triangulum II (Tri II) in Pan-STARRS images. Its luminosity $\left(450 L_{\odot}\right)$ and $2 \mathrm{D}$ halflight radius $(34 \mathrm{pc})$ are comparable to Segue 1 , the faintest galaxy known (Belokurov et al. 2007; Geha et al. 2009; Simon

\footnotetext{
* The data presented herein were obtained at the W. M. Keck Observatory, which is operated as a scientific partnership among the California Institute of Technology, the University of California and the National Aeronautics and Space Administration. The Observatory was made possible by the generous financial support of the W. M. Keck Foundation.
}

et al. 2011). Laevens et al. (2015a) suggested that Tri II could be associated with the Triangulum-Andromeda halo substructure (Majewski et al. 2004) or the Pan-Andromeda Archaeological Survey (PAndAS) stream (Martin et al. 2014). If so, then it could be one of the progenitors of that tidal debris. However, spatial coincidence is not sufficient evidence for the association. The velocity of the progenitor should also match that of the debris.

We obtained spectra of stars in Tri II in order to learn about its origin and identity. The velocity dispersion can identify it as a galaxy or a star cluster, and the mean velocity can support or disprove an association with stellar debris. We describe our observations in Section 2 and our measurements of velocities and metallicities in Section 3. We consider whether Tri II is in dynamical equilibrium or tidally disrupting in Section 4. Finally, we discuss the nature of Tri II and its importance for the study of dark matter in Section 5.

\section{OBSERVATIONS}

\subsection{Imaging}

We imaged Tri II with Keck/LRIS (Oke et al. 1995) on 2015 July 15 . We obtained simultaneous $10 \mathrm{~s}$ exposures with $V$ and $I$ filters in the blue and red channels, respectively. We also obtained $10 \mathrm{~s}$ exposures of the photometric standard field PG0231 in the same filters. We performed aperture photometry on both fields using SExtractor (Bertin \& Arnouts 1996). The photometric zeropoint was determined by finding the offsets between our instrumental magnitudes and P.B. Stetson's calibrated magnitudes in PG0231. ${ }^{4} \mathrm{We}$ discarded resolved galaxies by eliminating objects with class_star $<0.5$.

\footnotetext{
4 http://www.cadc-ccda.hia-iha.nrc-cnrc.gc.ca/en/community/STETSON/ standards/
} 

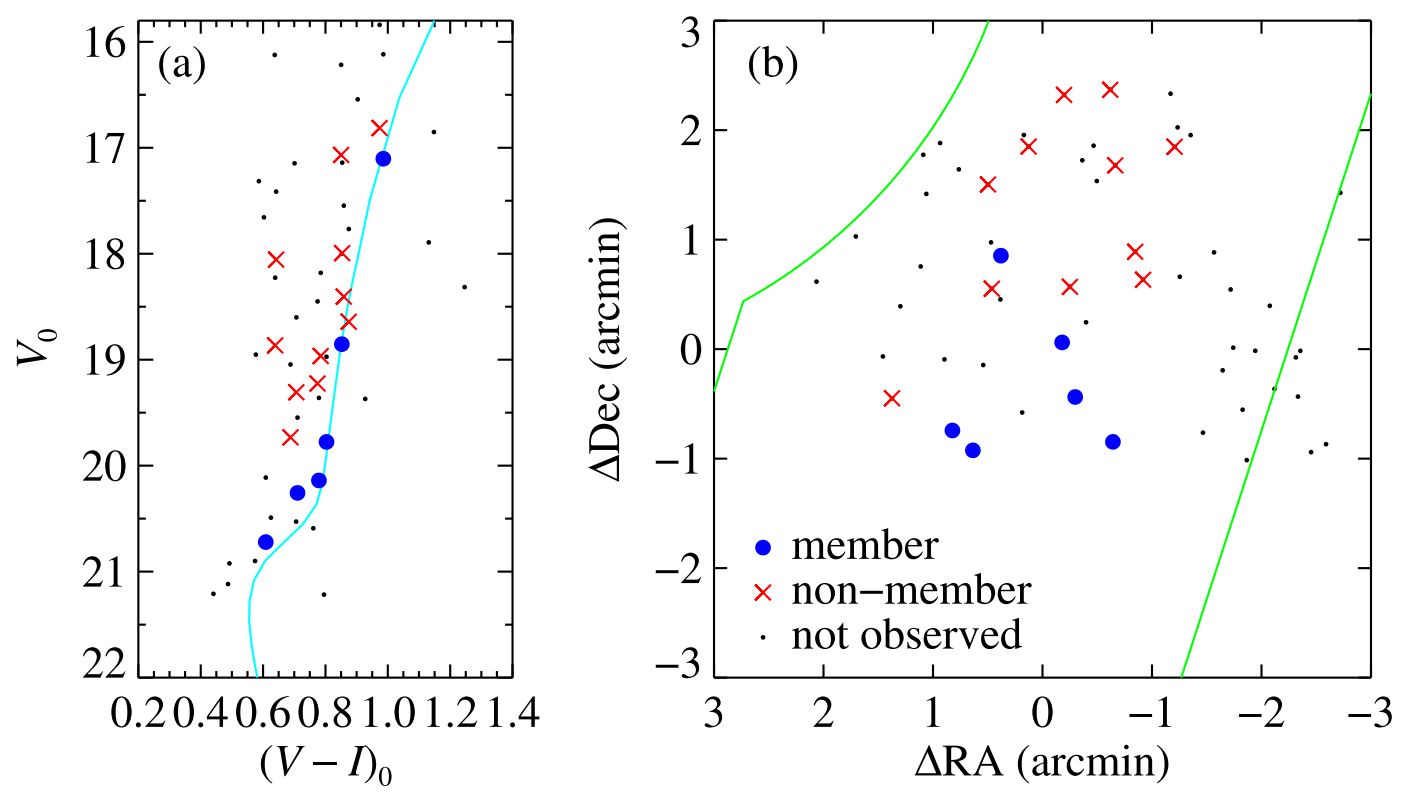

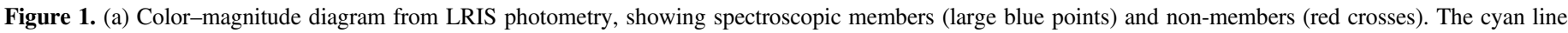

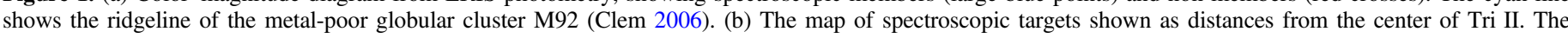

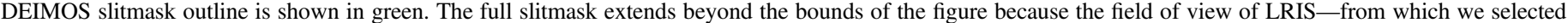
DEIMOS targets-is smaller than for DEIMOS.

\subsection{Spectroscopy}

We designed a slitmask for Keck/DEIMOS (Faber et al. 2003) from the LRIS photometry. We selected 19 stars for spectroscopy based on their locations in the colormagnitude diagram (CMD; Figure 1(a)). Stars near the subgiant and RGB tracks - the "ridgeline"-of the metal-poor globular cluster M92 (Clem 2006) were considered for spectroscopy. However, the field is dense enough that we could not target every star. When slitmask design constraints forced a choice among several stars, we chose to target the brightest star. Figure 1(b) shows the 17 spectroscopic targets with signal-to-noise ratio $(\mathrm{S} / \mathrm{N})$ sufficient for velocity measurements (see Section 3).

We obtained 61 minutes of DEIMOS exposures in 1."6 seeing on 2015 October 6 . The poor seeing resulted in low $\mathrm{S} / \mathrm{N}$ for the fainter stars. We obtained 52 minutes of exposures in 0 "' 9 seeing on 2015 October 7 . The spectra from the first night were used only to compare with velocity measurements from the second night (Section 3.1). The measurements of velocity and metallicity dispersions for Tri II are based only on data from 2015 October 7.

We reduced the data with the spec $2 \mathrm{~d}$ software (Cooper et al. 2012; Newman et al. 2013) with modifications described by Kirby et al. (2015a, 2015b). Among other improvements, the $2 \mathrm{D}$ wavelength solution was improved by tracing the sky lines along the slit, and the extraction was improved by taking into account differential atmospheric refraction along the slit.

\section{SPECTROSCOPIC MEASUREMENTS}

\subsection{Radial Velocities and Metallicities}

We measured heliocentric radial velocities ( $\left.v_{\text {helio }}\right)$ and metallicities $([\mathrm{Fe} / \mathrm{H}])$ in a manner identical to Kirby et al. (2015b), who based their analysis on Simon \& Geha (2007). Radial velocities were computed by finding the velocity that minimized the $\chi^{2}$ between the observed spectrum and eight template spectra observed with DEIMOS. The template spectrum with the lowest $\chi^{2}$ was used. We corrected the velocity shift due slit mis-centering by measuring the observed wavelength of telluric absorption in the stellar spectrum (e.g., Sohn et al. 2007). We computed errors due to random noise by finding the standard deviation of the velocities of $10^{3}$ Monte Carlo realizations of the spectrum. The total error, $\delta v$, was calculated by adding the random error in quadrature with a systematic error of $1.49 \mathrm{~km} \mathrm{~s}^{-1}$. The systematic error includes sources of uncertainty that cannot be attributed to random noise, such as uncorrected spectrograph flexure or small errors in the wavelength solution. The magnitude of the systematic error was calculated by Kirby et al. (2015b) by comparing repeated measurements of the same stars.

We tested our estimate of velocity errors by comparing the low-S/N measurements of $v_{\text {helio }}$ from 2015 October 6 to the high-S/N measurements from 2015 October 7 . Of the six stars we determined to be members (Section 3.2), five velocities were measurable with data from the first night. We computed $\left(v_{\text {helio,1 }}-v_{\text {helio, } 2}\right) / \sqrt{\delta v_{1}^{2}+\delta v_{2}^{2}}$ for each pair of measurements of the member stars. The variance of this quantity should be 1 if we estimated errors properly. We measured it to be $1.3 \pm 0.6$. Hence, the error estimates are reasonable.

We measured effective temperatures $\left(T_{\text {eff }}\right)$ and metallicities for member stars with sufficient $\mathrm{S} / \mathrm{N}$ in the same manner as Kirby et al. (2008, 2010). First, we divided the spectrum by a polynomial fit to regions of the spectrum free of absorption lines. Next, we estimated temperatures and surface gravities $(\log g)$ by fitting Yonsei-Yale theoretical isochrones (Demarque et al. 2004) to observed stellar colors and magnitudes. Then, we used these parameters and an initial guess of $[\mathrm{Fe} / \mathrm{H}]=-1.5$ to construct a synthetic spectrum at the observed spectrum's resolution. This spectrum was linearly interpolated from Kirby et al.'s (2010) synthetic spectral grid. In order to minimize $\chi^{2}$ between the observed and synthetic spectra, we changed the synthetic spectrum's $T_{\text {eff }}$ and $[\mathrm{Fe} / \mathrm{H}]$ but held $\log g$ fixed. The measured values of $T_{\text {eff }}$ and $[\mathrm{Fe} / \mathrm{H}]$ 
Table 1

Target List

\begin{tabular}{|c|c|c|c|c|c|c|c|c|c|c|}
\hline ID & R.A. (J2000) & Decl. (J2000) & $\begin{array}{c}V_{0} \\
(\mathrm{mag})\end{array}$ & $\begin{array}{c}(V-I)_{0} \\
(\mathrm{mag})\end{array}$ & $\begin{array}{l}\mathrm{S} / \mathrm{N}^{\mathrm{a}} \\
\left(\AA^{-1}\right)\end{array}$ & $\begin{array}{c}v_{\text {helio }} \\
\left(\mathrm{km} \mathrm{s}^{-1}\right)\end{array}$ & Member? & $\begin{array}{l}T_{\text {eff }} \\
(\mathrm{K})\end{array}$ & $\begin{array}{c}\log g \\
\left(\mathrm{~cm} \mathrm{~s}^{-2}\right)\end{array}$ & {$[\mathrm{Fe} / \mathrm{H}]$} \\
\hline 166 & 021311.42 & +361233.0 & 17.99 & 0.85 & 115 & $11.0 \pm 5.9$ & $\mathrm{~N}$ & $\ldots$ & $\ldots$ & $\ldots$ \\
\hline 174 & 021312.85 & +361120.2 & 18.06 & 0.64 & 102 & $-100.8 \pm 1.6$ & $\mathrm{~N}$ & $\ldots$ & $\ldots$ & $\ldots$ \\
\hline 177 & $02 \quad 1313.21$ & +361135.5 & 19.73 & 0.69 & 36 & $-280.7 \pm 2.5$ & $\mathrm{~N}$ & $\ldots$ & $\ldots$ & $\ldots$ \\
\hline 126 & 021314.11 & +361222.9 & 16.81 & 0.97 & 127 & $2.4 \pm 1.5$ & $\mathrm{~N}$ & $\ldots$ & $\ldots$ & $\ldots$ \\
\hline 128 & $02 \quad 1314.21$ & +360951.4 & 19.78 & 0.80 & 24 & $-384.9 \pm 3.2$ & $\mathrm{Y}$ & 5292 & 3.17 & $-2.72 \pm 0.39$ \\
\hline 127 & $0213 \quad 14.33$ & +361304.3 & 19.31 & 0.71 & 54 & $-86.0 \pm 1.8$ & $\mathrm{~N}$ & $\ldots$ & $\ldots$ & $\ldots$ \\
\hline 116 & 021315.92 & +361016.0 & 20.26 & 0.71 & 26 & $-377.6 \pm 3.7$ & $\mathrm{Y}$ & $\ldots$ & $\ldots$ & $\ldots$ \\
\hline 113 & 021316.16 & +361116.3 & 18.97 & 0.78 & 72 & $-57.6 \pm 1.6$ & $\mathrm{~N}$ & $\ldots$ & $\ldots$ & $\ldots$ \\
\hline 111 & 021316.42 & +361301.5 & 18.86 & 0.64 & 66 & $-62.0 \pm 1.9$ & $\mathrm{~N}$ & $\ldots$ & $\ldots$ & $\ldots$ \\
\hline 106 & 021316.51 & +361045.9 & 17.10 & 0.99 & 219 & $-382.3 \pm 1.5$ & $\mathrm{Y}$ & 4922 & 1.88 & $-2.86 \pm 0.11$ \\
\hline 100 & $02 \quad 13 \quad 18.03$ & +361233.2 & 18.64 & 0.87 & 87 & $-38.0 \pm 1.6$ & $\mathrm{~N}$ & $\ldots$ & $\ldots$ & $\ldots$ \\
\hline 91 & 021319.28 & +361133.4 & 20.14 & 0.78 & 29 & $-386.0 \pm 3.1$ & $\mathrm{Y}$ & $\ldots$ & $\ldots$ & $\ldots$ \\
\hline 84 & 021319.69 & +361115.3 & 19.22 & 0.77 & 60 & $-66.4 \pm 1.7$ & $\mathrm{~N}$ & $\ldots$ & $\ldots$ & $\ldots$ \\
\hline 82 & 021319.87 & +361212.4 & 18.41 & 0.86 & 101 & $-177.0 \pm 1.6$ & $\mathrm{~N}$ & $\ldots$ & $\ldots$ & $\ldots$ \\
\hline 76 & 021320.55 & +360946.7 & 20.72 & 0.61 & 17 & $-389.7 \pm 3.0$ & $\mathrm{Y}$ & $\ldots$ & $\ldots$ & $\ldots$ \\
\hline 65 & 021321.48 & +360957.6 & 18.85 & 0.85 & 81 & $-374.5 \pm 1.7$ & $\mathrm{Y}$ & 5169 & 2.74 & $-2.04 \pm 0.13$ \\
\hline 45 & 021324.21 & +361015.3 & 17.07 & 0.85 & 152 & $-62.1 \pm 1.5$ & $\mathrm{~N}$ & $\ldots$ & $\ldots$ & $\ldots$ \\
\hline
\end{tabular}

Note.

${ }^{\mathrm{a}}$ To convert to $\mathrm{S} / \mathrm{N}$ per pixel, multiply by 0.57 .

are those of the synthetic spectrum with the minimum $\chi^{2}$. The error on $[\mathrm{Fe} / \mathrm{H}]$ is the appropriate diagonal term of the covariance matrix added in quadrature with a systematic error of 0.11 dex, which Kirby et al. (2010) determined from repeat measurements. We kept $[\mathrm{Fe} / \mathrm{H}]$ measurements of the three stars with uncertainties less than $0.5 \mathrm{dex}$ and discarded the others.

These three stars lie in the range $-3<[\mathrm{Fe} / \mathrm{H}]<-2$ with a mean of $\langle[\mathrm{Fe} / \mathrm{H}]\rangle=-2.50 \pm 0.08$. Tri II has the lowest measured mean metallicity of any galaxy except Segue 1 (Frebel et al. 2014) and Reticulum II (Simon et al. 2015; Walker et al. 2015). However, the metallicity measurements for Tri II are based on only three stars. While the standard error of the mean is 0.08 dex, the mean metallicity of a larger sample could be substantially different.

Table 1 lists the radial velocities for all the stars we observed with DEIMOS except two stars with spectra that were too noisy to identify any absorption lines. Temperatures and metallicities are given for the three member stars with sufficient quality for those measurements.

\subsection{Membership and Velocity Dispersion}

We identified a peak in the velocity distribution of the observed stars around $-380 \mathrm{~km} \mathrm{~s}^{-1}$. We took stars within $50 \mathrm{~km} \mathrm{~s}^{-1}$ of this peak as the initial member list. We measured the velocity dispersion $\left(\sigma_{v}\right)$ of these six stars in the same manner as Kirby et al. (2014, 2015b), who based their analysis on Walker et al. (2006). We estimated $\sigma_{v}$ via maximum likelihood. A Monte Carlo Markov chain (MCMC) with $10^{7}$ trials explored the parameter space of mean velocity $\left(\left\langle v_{\text {helio }}\right\rangle\right)$ and $\sigma_{v}$. We quote the values corresponding to the peaks of the probability distributions as the measurements of $\left\langle v_{\text {helio }}\right\rangle$ and $\sigma_{v}$. The asymmetric $1 \sigma$ confidence interval on $\sigma_{v}$ is the range on either side of the mean value that bounds $68.3 \%$ of the trials.

We measured $\left\langle v_{\text {helio }}\right\rangle=-382.1 \pm 2.9 \mathrm{~km} \mathrm{~s}^{-1}$ and $\sigma_{v}=5.1_{-1.4}^{+4.0} \mathrm{~km} \mathrm{~s}^{-1}$. All six candidate member stars are within $1.1 \sigma_{v}$ of $\left\langle v_{\text {helio }}\right\rangle$. Furthermore, all six stars are close to the M92 ridgeline in Figure 1(a), indicating that they pass a CMD membership cut. None of the six stars shows a strong
Na I 8190 doublet, which would have indicated that the star is a foreground dwarf.

Figure 2 shows the spectra of the six member stars in observed wavelength. Ca II 8542 appears at a different observed wavelength for every star, showing that we have resolved the velocity dispersion of Tri II.

Other than Tri II, only three galaxies with $L<10^{4} L_{\odot}$ have published measurements of $\sigma_{v}>5 \mathrm{~km} \mathrm{~s}^{-1}$ : Boötes II (Koch et al. 2009), Pisces II (Kirby et al. 2015b), and Ursa Major II (Simon \& Geha 2007). Koch et al. measured $\sigma_{v}=10.5 \pm 7.4 \mathrm{~km} \mathrm{~s}^{-1}$ for Boötes II, but more recent measurements have found a smaller dispersion (M. Geha et al. 2015 , in preparation) and the presence of at least one binary that inflates the apparent dispersion ( $\mathrm{Ji}$ et al. 2015).

Even if all of the stars are members, some of them might be binaries. The orbital velocity of the binary would artificially inflate our measurement of $\sigma_{v}$ for the galaxy. We tested the robustness of our measurement of $\sigma_{v}$ by jackknife resampling. We recalculated $\sigma_{v}$ for each of the six subsets of member stars formed by removing one star. All of the probability distributions are well separated from zero. The minimum velocity dispersion, calculated by removing star 65 , is $2.8_{-1.7}^{+4.0} \mathrm{~km} \mathrm{~s}^{-1}$. The jackknife error, calculated as the standard deviation of $\sigma_{v}$ for all of the jackknife trials, is $1.2 \mathrm{~km} \mathrm{~s}^{-1}$, somewhat smaller than the error calculated from the MCMC distribution.

\section{DYNAMICAL EQUILIBRIUM}

Table 2 gives some characteristics of Tri II, including the mass within the 3D half-light radius $\left(M_{1 / 2}\right.$; Wolf et al. 2010). This quantity and its associated quantities, mass-to-light ratio $\left[\left(M / L_{V}\right)_{1 / 2}\right]$ and density $\left(\rho_{1 / 2}\right)$ within the 3D half-light radius, presume that the galaxy is spherically symmetric and in dynamical equilibrium. However, the velocity dispersion accurately reflects the mass even in the presence of moderate tidal forces (Oh et al. 1995). If the velocities of the stars we measured are very heavily affected by tides, then these quantities are not meaningful. We now consider whether the center of Tri II is in dynamical equilibrium. 


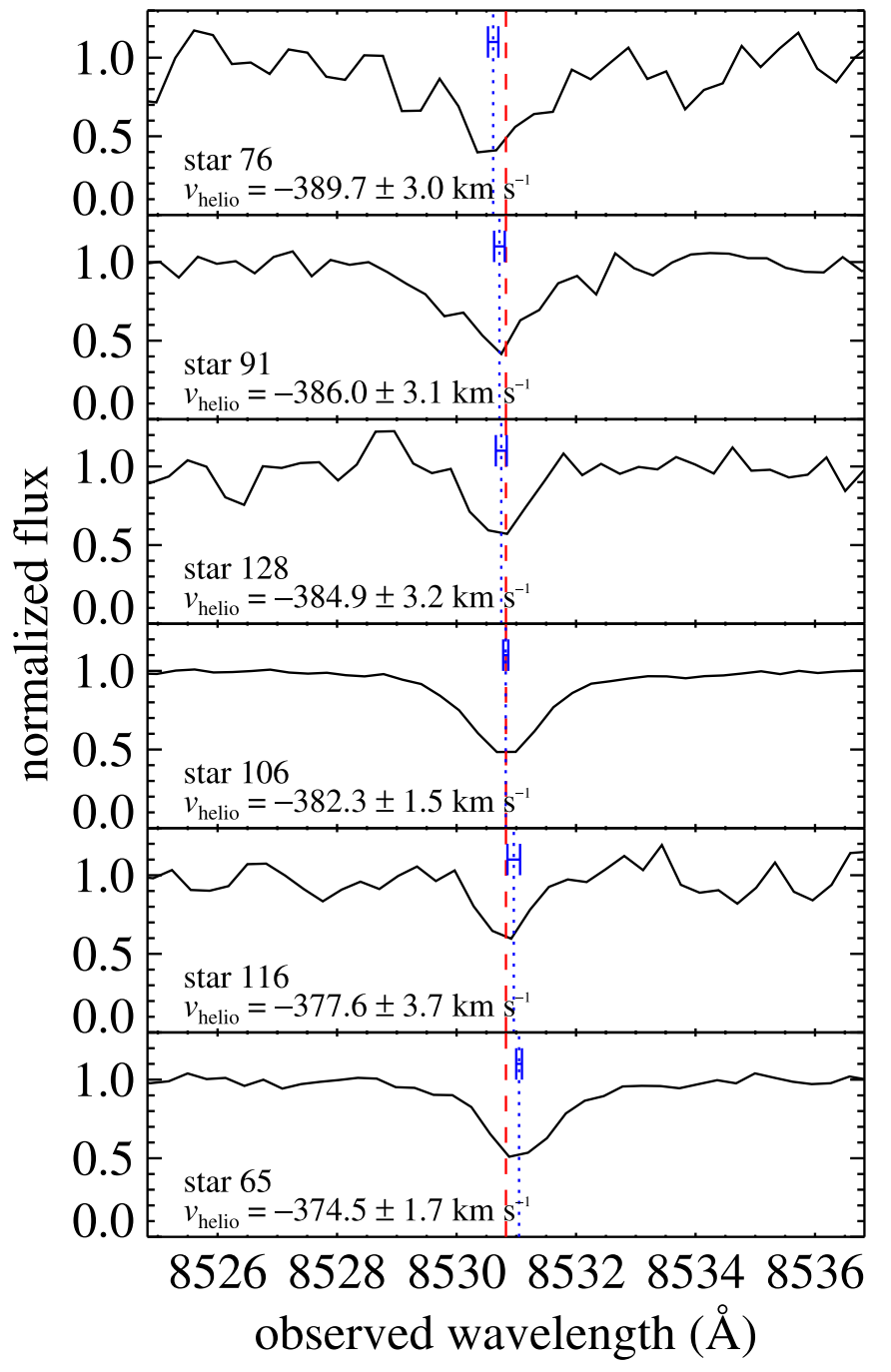

Figure 2. Small regions of DEIMOS spectra of the six member stars shown against observed wavelength. The full spectrum - much wider than shown here -is used for the velocity measurement. The dashed red line shows the observed wavelength of Ca II 8542 at the mean geocentric velocity of Tri II. The blue dotted lines show the observed wavelength of $\mathrm{Ca}$ II 8542 for each star, and the blue whiskers indicate the $\pm 1 \sigma$ uncertainty of the observed centroid of the absorption line for each star. The spectra are ordered from the lowest to highest radial velocity. The shift of Ca II 8542 is apparent even by eye.

The MW exerts the maximum tidal shear on satellite galaxies at their pericenters (e.g., Mayer et al. 2001). It is more likely to find a tidally disrupting galaxy close to the Galactic center than far from it. Tri II is only $D_{\mathrm{GC}}=36 \pm 2 \mathrm{kpc}$ from the Galactic center (Laevens et al. 2015b). It is also rapidly approaching its pericenter. Assuming a solar orbital velocity of $220 \mathrm{~km} \mathrm{~s}^{-1}$, the velocity of Tri II relative to the Galactic standard of rest (GSR) is $-262 \mathrm{~km} \mathrm{~s}^{-1}$. The fact that Tri II is approaching pericenter rather than receding from it is consistent with its imminent tidal disruption. However, its large velocity limits the time that it will spend near pericenter and consequently reduces the total tidal effect.

A tidally disrupting galaxy could have a high ellipticity. The ellipticity of Tri II is $\epsilon=0.21_{-0.21}^{+0.17}$ (Laevens et al. 2015b). In contrast with presently disrupting galaxies, like Sagittarius $(\epsilon=0.65$; Majewski et al. 2003), Tri II is not obviously elliptical.
Table 2

Properties of Triangulum II

\begin{tabular}{lc}
\hline \hline Property & Value \\
\hline$N_{\text {member }}$ & 6 \\
$\log \left(L_{V} / L_{\odot}\right)$ & $2.65 \pm 0.20$ \\
$r_{h}$ & $3.9_{-0.9}^{+1.1} \operatorname{arcmin}$ \\
$r_{h}$ & $34_{-8}^{+9} \mathrm{pc}$ \\
$\left\langle v_{\text {helio }}\right\rangle$ & $-382.1 \pm 2.9 \mathrm{~km} \mathrm{~s}^{-1}$ \\
$v_{\mathrm{GSR}}$ & $-262 \mathrm{~km} \mathrm{~s}^{-1}$ \\
$\sigma_{v}$ & $5.1_{-1.4}^{+4.0} \mathrm{~km} \mathrm{~s}^{-1}$ \\
$\log \left(M_{1 / 2} / M_{\odot}\right)^{\mathrm{a}}$ & $5.9_{-0.2}^{+0.4}$ \\
$\left(M / L_{V}\right)_{1 / 2}{ }^{\mathrm{a}, \mathrm{b}}$ & $3600_{-2100}^{+3500} M_{\odot} L_{\odot}^{-1}$ \\
$\rho_{1 / 2}{ }^{\mathrm{a}, \mathrm{c}}$ & $4.8_{-3.5}^{+8.1} M_{\odot} \mathrm{pc}^{-3}$ \\
$\langle[\mathrm{Fe} / \mathrm{H}]\rangle$ & $-2.50 \pm 0.08$ \\
\hline
\end{tabular}

Notes.

a These quantities presume that Tri II is in dynamical equilibrium.

b Mass-to-light ratio within the half-light radius, calculated as $M_{1 / 2}=4 G^{-1} \sigma_{v}^{2} r_{h}$ (Wolf et al. 2010).

${ }^{c}$ Density within the half-light radius.

References. The measurements of $\log L_{V}$ and $r_{h}$ come from Laevens et al. (2015b).

Along with high ellipticity, ongoing tidal disruption could cause a non-Gaussian velocity distribution. A Shapiro-Wilk test gives a $p$ value of 0.87 . A completely Gaussian distribution would have a $p$ value of 1 . Therefore, there is no evidence for non-Gaussianity in the velocity distribution. Of course, our small sample size limits the significance of this result. Furthermore, we have measured only line of sight velocities, and even a tidally disrupting system may have normally distributed velocities along some lines of sight.

We estimated a lower limit to the tidal radius assuming that $M_{1 / 2}$ is the entire mass of Tri II. The Roche limit for a fluid satellite is $r_{\text {tidal }} \sim 0.4 D_{\mathrm{GC}}\left(M_{1 / 2} / M_{\mathrm{MW}}\right)^{1 / 3}$, where $M_{\mathrm{MW}} \approx 10^{12} M_{\odot}$ is the MW's mass. Under these assumptions, $r_{\text {tidal }} \approx 140 \mathrm{pc}$ for Tri II, or about three times its 3D half-light radius ( $4 / 3$ of the $2 \mathrm{D}$, projected half-light radius). The tidal radius would shrink to the same value as the $3 \mathrm{D}$ half-light radius at $\sim 12 \mathrm{kpc}$ from the Galactic center. Therefore, all of the stars we observed in Tri II are presently insulated from Galactic tides. Although this estimate of tidal radius presumes that the velocity dispersion reflects the present mass, simulations suggest that the velocity dispersion is a good indicator of the instantaneous mass except for a short time after pericenter, even for systems experiencing significant tidal stripping (Oh et al. 1995; Muñoz et al. 2008; Peñarrubia et al. 2009).

Laevens et al. (2015b) noted the possible association of Tri II with the Triangulum-Andromeda halo substructure (Majewski et al. 2004) or the PAndAS stream (Martin et al. 2014). Association with such halo debris might indicate that the galaxy is being disrupted and that it is the source of the debris. However, Deason et al. (2014) measured the GSR velocities of these structures as $30-70 \mathrm{~km} \mathrm{~s}^{-1}$, which is roughly $300 \mathrm{~km} \mathrm{~s}^{-1}$ different from $v_{\mathrm{GSR}}$ for Tri II. Therefore, there is no presently known stream that could be associated with Tri II. This does not prove that Tri II is in dynamical equilibrium, but it does show that, if Tri II is being tidally disrupted, it is not the source of the Triangulum-Andromeda or PAndAS stellar debris.

The luminosity-metallicity relation (LZR; Figure 3(a)) is a diagnostic of tidal stripping. The LZR for classical dwarf 


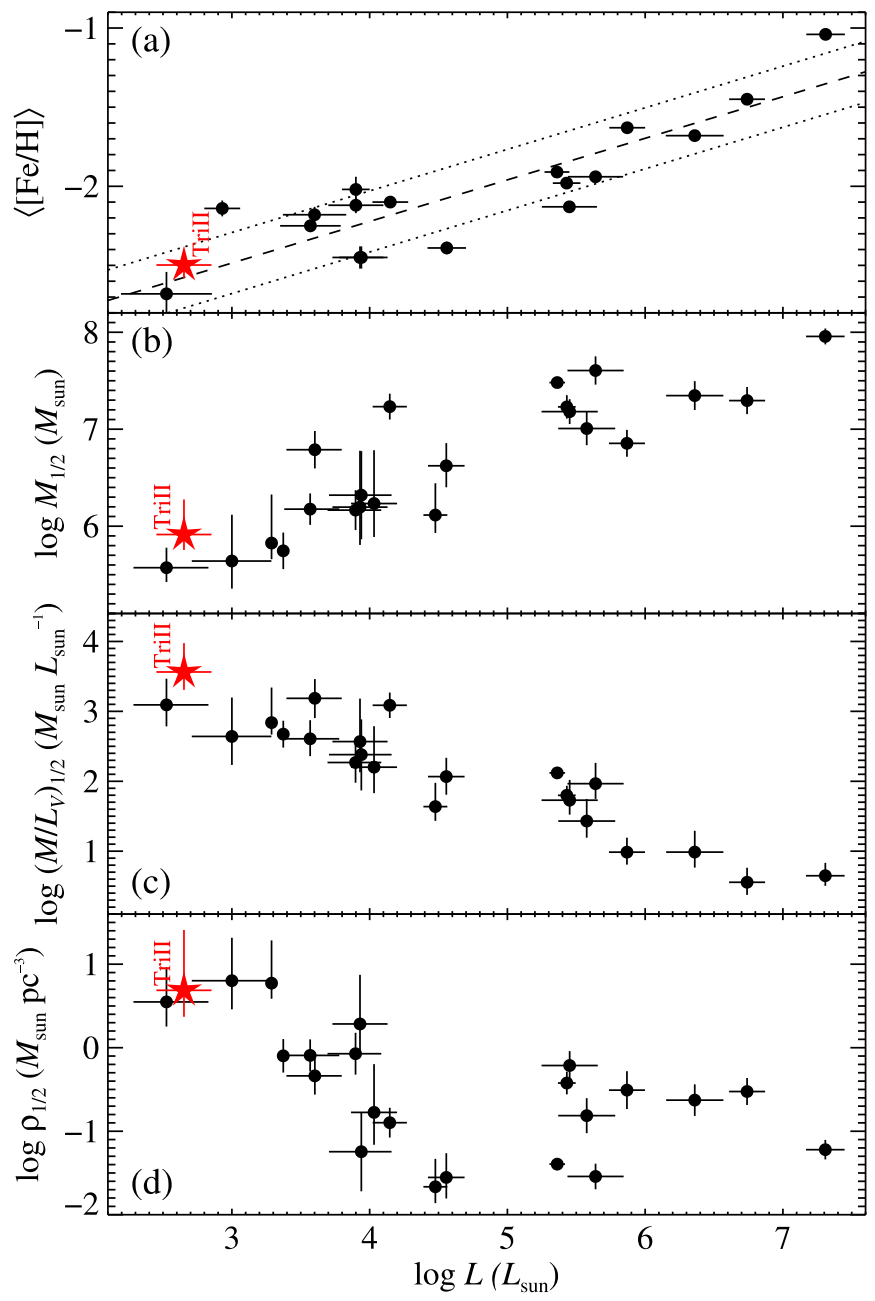

Figure 3. (a) Luminosity-metallicity relation for MW satellite galaxies. The dashed line shows the linear fit to the galaxies except Tri II (Kirby et al. 2013b, 2015b; Frebel et al. 2014), and the dotted lines show the rms dispersion about the fit. (b) Masses of MW satellite galaxies within their 3D half-light radii assuming dynamical equilibrium. Data are from (McConnachie 2012 and references therein), Simon et al. (2015), Koposov et al. (2015), and Kirby et al. (2015b). (c) Mass-to-light ratios and (d) densities within the 3D half-light radii. Boötes II and III are not shown because their published velocity dispersions do not reflect their dynamical masses.

galaxies is very tight, with an rms of only 0.13 dex (Kirby et al. 2011; Kirby et al. 2013b). If a galaxy initially conforms to the LZR, then tidal stripping will decrease its luminosity while keeping its average metallicity roughly constant. This corresponds to a leftward move in Figure 3(a). Some of the galaxies with $L \lesssim 10^{4} L_{\odot}$, especially Segue 2 (Kirby et al. 2013a), lie significantly to the left of the LZR. On the other hand, Tri II is consistent with the LZR. Therefore, any tidal stripping that already happened is likely to have been mild.

\section{DISCUSSION}

Tri II satisfies the definition of "galaxy" given by Willman \& Strader (2012). The velocity dispersion is much too large to be explained by stars alone. We also found a large dispersion in metallicity. The stars span $0.8 \mathrm{dex}$ in $[\mathrm{Fe} / \mathrm{H}]$, which is evidence for chemical self-enrichment. The present mass of stars alone would not have been enough to retain supernova ejecta. Hence, without substantial mass loss, the velocity and metallicity dispersions are evidence for a large amount of dark matter.
It is unclear whether Tri II is in dynamical equilibrium. With a total luminosity of only $450 L_{\odot}$, the galaxy has very few stars available to measure its shape very precisely. Even fewer stars are available for spectroscopy. Therefore, resolving this question will be very difficult. Regardless, we now consider Tri II's place among the MW satellite population under the presumption of dynamical equilibrium.

Figures 3(b)-(d) show the trends of $M_{1 / 2},\left(M / L_{V}\right)_{1 / 2}$, and $\rho_{1 / 2}$ with luminosity. Tri II has the largest mass-to-light ratio $\left(3600_{-2100}^{+3500} M_{\odot} L_{\odot}^{-1}\right)$ of any galaxy except Boötes III, whose tidal disruption is nearly complete (Carlin et al. 2009; Grillmair 2009). If Tri II is in dynamical equilibrium, then it is the most dark-matter dominated galaxy known.

The five galaxies with $\rho_{1 / 2}>1 M_{\odot} \mathrm{pc}^{-3}$ in order from densest to least dense are Willman 1, Horologium I, Tri II, Segue 1, and Pisces II. Four of these galaxies comprise the least luminous galaxies with measured velocity dispersions. This correlation could arise because these galaxies also have the smallest half-light radii. If galaxies' mass profiles peak in the center, then smaller galaxies will be observed to have larger $\rho_{1 / 2}$, even if their total masses and mass profiles are identical. The correlation between $\rho_{1 / 2}$ and $L_{V}$ could also arise because less massive galaxies are more susceptible to tidal stripping. Hence, the measurement of $\rho_{1 / 2}$ would be invalid because the galaxies are not in equilibrium. Willman 1 has a velocity distribution that does not seem consistent with dynamical equilibrium (Willman et al. 2011). The velocity dispersions of Horologium I, Pisces II, and Tri II were all measured from 5-7 stars (Kirby et al. 2015b; Koposov et al. 2015, and this work). Hence, Segue 1 (Simon et al. 2011) remains the galaxy with the most secure measurement of a very high central density.

In summary, we measured $\sigma_{v}=5.1_{-1.4}^{+4.0} \mathrm{~km} \mathrm{~s}^{-1}$ for Tri II. The present measurements cannot determine whether the galaxy is in dynamical equilibrium or being tidally disrupted. However, the possibility that it is in equilibrium is very exciting. Tri II would be the most dark-matter dominated galaxy known, and it would be an excellent candidate for the indirect, gamma-ray detection of dark matter annihilation. The annihilation signal scales as $\rho^{2}$, which makes very dense galaxies-possibly including Tri II-the best prospects for detection.

We thank Gina Duggan for obtaining LRIS images, Emily Cunningham for helpful statistics advice, and the anonymous referee for helpful feedback. P.G. acknowledges support from NSF grants AST-1010039 and AST-1412648. We are grateful to the many people who have worked to make the Keck Telescope and its instruments a reality and to operate and maintain the Keck Observatory. The authors wish to extend special thanks to those of Hawaiian ancestry on whose sacred mountain we are privileged to be guests. Without their generous hospitality, none of the observations presented herein would have been possible.

Facilities: Keck:I (LRIS), Keck:II (DEIMOS).

\section{REFERENCES}

Abazajian, K. N., Adelman-McCarthy, J. K., Agüeros, M. A., et al. 2009, ApJS, 182, 543

Bechtol, K., Drlica-Wagner, A., Balbinot, E., et al. 2015, ApJ, 807, 50 Belokurov, V., Zucker, D. B., Evans, N. W., et al. 2007, ApJ, 654, 897 Bertin, E., \& Arnouts, S. 1996, A\&AS, 117, 393 
Carlin, J. L., Grillmair, C. J., Muñoz, R. R., Nidever, D. L., \& Majewski, S. R. 2009, ApJL, 702, L9

Clem, J. L. 2006, PhD thesis, Univ. Victoria

Cooper, M. C., Newman, J. A., Davis, M., Finkbeiner, D. P., \& Gerke, B. F. 2012, Astrophysics Source Code Library, ascl:1203.003

Deason, A. J., Belokurov, V., Hamren, K. M., et al. 2014, MNRAS, 444, 3975

Demarque, P., Woo, J.-H., Kim, Y.-C., \& Yi, S. K. 2004, ApJS, 155, 667

Faber, S. M., Phillips, A. C., Kibrick, R. I., et al. 2003, Proc. SPIE, 4841, 1657

Flaugher, B. L., Abbott, T. M. C., Angstadt, R., et al. 2012, Proc. SPIE, 8446, 844611

Frebel, A., Simon, J. D., \& Kirby, E. N. 2014, ApJ, 786, 74

Geha, M., Willman, B., Simon, J. D., et al. 2009, ApJ, 692, 1464

Grillmair, C. J. 2009, ApJ, 693, 1118

Ji, A. P., Frebel, A., Simon, J. D., \& Geha, M. 2015, ApJ, submitted (arXiv:1510.07632)

Kaiser, N., Burgett, W., Chambers, K., et al. 2010, Proc. SPIE, 7733, 77330E

Kim, D., \& Jerjen, H. 2015, ApJL, 808, L39

Kirby, E. N., Boylan-Kolchin, M., Cohen, J. G., et al. 2013a, ApJ, 770, 16

Kirby, E. N., Bullock, J. S., Boylan-Kolchin, M., Kaplinghat, M., \& Cohen, J. G. 2014, MNRAS, 439, 1015

Kirby, E. N., Cohen, J. G., Guhathakurta, P., et al. 2013b, ApJ, 779, 102

Kirby, E. N., Guhathakurta, P., Simon, J. D., et al. 2010, ApJS, 191, 352

Kirby, E. N., Guhathakurta, P., \& Sneden, C. 2008, ApJ, 682, 1217

Kirby, E. N., Guo, M., Zhang, A. J., et al. 2015a, ApJ, 801, 125

Kirby, E. N., Lanfranchi, G. A., Simon, J. D., Cohen, J. G., \& Guhathakurta, P. 2011, ApJ, 727, 78

Kirby, E. N., Simon, J. D., \& Cohen, J. G. 2015b, ApJ, 810, 56
Koch, A., Wilkinson, M. I., Kleyna, J. T., et al. 2009, ApJ, 690, 453

Koposov, S. E., Casey, A. R., Belokurov, V., et al. 2015, ApJ, 811, 62

Laevens, B. P. M., Martin, N. F., Bernard, E. J., et al. 2015a, ApJ, 813, 44

Laevens, B. P. M., Martin, N. F., Ibata, R. A., et al. 2015b, ApJL, 802, L18

Majewski, S. R., Ostheimer, J. C., Rocha-Pinto, H. J., et al. 2004, ApJ, 615,738

Majewski, S. R., Skrutskie, M. F., Weinberg, M. D., \& Ostheimer, J. C. 2003, ApJ, 599, 1082

Martin, N. F., Geha, M., Ibata, R. A., et al. 2015, ApJL, submitted (arXiv:1510.01326)

Martin, N. F., Ibata, R. A., Rich, R. M., et al. 2014, ApJ, 787, 19

Mayer, L., Governato, F., Colpi, M., et al. 2001, ApJ, 559, 754

McConnachie, A. W. 2012, AJ, 144, 4

Muñoz, R. R., Majewski, S. R., \& Johnston, K. V. 2008, ApJ, 679, 346

Newman, J. A., Cooper, M. C., Davis, M., et al. 2013, ApJS, 208, 5

Oh, K. S., Lin, D. N. C., \& Aarseth, S. J. 1995, ApJ, 442, 142

Oke, J. B., Cohen, J. G., Carr, M., et al. 1995, PASP, 107, 375

Peñarrubia, J., Navarro, J. F., McConnachie, A. W., \& Martin, N. F. 2009, ApJ, 698, 222

Simon, J. D., Drlica-Wagner, A., Li, T. S., et al. 2015, ApJ, 808, 95

Simon, J. D., \& Geha, M. 2007, ApJ, 670, 313

Simon, J. D., Geha, M., Minor, Q. E., et al. 2011, ApJ, 733, 46

Sohn, S. T., Majewski, S. R., Muñoz, R. R., et al. 2007, ApJ, 663, 960

Walker, M. G., Mateo, M., Olszewski, E. W., et al. 2006, AJ, 131, 2114

Walker, M. G., Mateo, M., Olszewski, E. W., et al. 2015, ApJ, 808, 108

Willman, B., Geha, M., Strader, J., et al. 2011, AJ, 142, 128

Willman, B., \& Strader, J. 2012, AJ, 144, 76

Wolf, J., Martinez, G. D., Bullock, J. S., et al. 2010, MNRAS, 406, 1220 